\title{
A NEW 2D INTEGRABLE SYSTEM WITH A QUARTIC SECOND INVARIANT
}

\author{
Yehia Hamad M. \\ Department of Mathematics, Faculty of Science, \\ Mansoura University, Mansoura 35516, Egypt \\ Email: hyehia@mans.edu.eg
}

August 13, 2018

\begin{abstract}
The construction of all 2-D Lagrangian systems, which admits besides the energy another integral of motion that is quartic in velocities was reduced in our previous article (J. Phys. A: Math. Gen., 39, 5807-5824, 2006) to a single nonlinear PDE.

In the present note we introduce a new solution of this equation, leading to a new integrable system with a quartic integral, which involves 16 free parameters. A special case of the new system admits interpretation in a problem of rigid body dynamics. It gives a new integrable variation of the cases due to Kovalevskaya (1889), Chaplygin (1903) Goriatchev (1916) and Yehia (2006).
\end{abstract}

\section{Introduction}

\section{$1.1 \quad$ Historical}

The famous Kovalevskaya's integrable case of rigid body dynamics was the first example of a mechanical system that admits an integral of motion quartic in velocities [1. For more than a century this case attracted attention of many specialists, who treated explicit solution in terms of time and gave several modifications and generalizations. Only in the last three decades there appeared a few new integrable systems with a quartic integral, but mainly concerning the motion of a particle in the Euclidean plane under the action of certain potential forces. A short, but nearly complete up to its date, list of those systems can be found in Hietarinta's review paper [2]. A few cases of the same type were obtained later in [3].

In virtue of Maupertuis principle, the motion of a natural mechanical system can be brought into equivalence (in the orbital sense) with the geodesic flow on some Riemannian metric. Metrics on the Riemannian sphere associated with 
known integrable cases of rigid body dynamics were constructed in [4. Two families of integrable systems with a quartic integral on $S^{2}$ were obtained in [5] and [6]. Few more works discussed possible integrable systems with low-degree polynomials on $S^{2}$ and the hyperbolic plane $H^{2}$ (see e.g. [7-[9]).

The method introduced in our work [10] and developed in several later works, has led to construction of a large number of several-parameter families of integrable systems with a complementary integral ranging from second to fourth degree (see e.g. [14, 15]). This method leads in a natural way to severalparameter integrable systems that allow as special cases, for particular values of the parameters, interpretations as motion on various flat and curved 2-D manifolds. Although our primary interest is in systems on Riemannian manifolds, some of the constructed integrable systems live on pseudo-Euclidean or pseudoRiemannian manifolds. New examples of integrable rigid body dynamics are common by-products of this method [10]-17].

The culmination of this method was the construction of the so-called "master" system with a quartic integral [16. It involves the largest ever number of 21 parameters and covers almost all systems of that type that were known earlier. Applied to rigid body dynamics, this system gave rise to 4 new integrable cases of motion under action of potential forces. In two cases the potential is a single valued function on the configuration space, but multivalued in the other two [11.

The problem of constructing all integrable mechanical systems admitting a quartic complementary integral on a Riemannian manifold has been reduced in [16] to a single nonlinear PDE, which we call the resolving equation. A solution of this equation is what we call generating function. This generates a conservative lagrangian system together with its quartic integral, valid on its zero-energy level. In the present article we introduce a new solution of the resolving equation. We also formulate a theorem that has been implicitly used in our previous work to construct systems integrable on arbitrary energy levels from condtional ones usually built on the zero-energy level. In its final unconditional form the integrable system based on the new solution involves 15 parameters. Special cases of it introduce new integrable cases of the types investigated earlier by Toda, Bozis and others for a particle moving in the Euclidean plane. A special case of the new system adds a rare new integrable problem in rigid body dynamics.

\subsection{Construction of integrable systems}

According to a result of Birkhoff [18, the general natural mechanical system (on an arbitrary 2D Riemannian configuration space) can always be reduced in certain (isometric) coordinates $\xi, \eta$ and time parametrization $\tau$ to the form of a ficticious plane system described by the Lagrangian

$$
L=\frac{1}{2}\left[\xi^{\prime 2}+\eta^{\prime 2}\right]+U, U=U(\xi, \eta)
$$


so that the equations of motion become

$$
x^{\prime \prime}=\frac{\partial U}{\partial x} \quad y^{\prime \prime}=\frac{\partial U}{\partial y}
$$

are restricted to their zero-energy level

$$
\xi^{\prime 2}+\eta^{2}-2 U=0
$$

The energy constant $h$ for the original system enters linearly as a parameter in the ficticious force function $U$, which has the structure

$$
U=\Lambda(h-V)
$$

where $V$ is the potential of the original system and $\Lambda$ is a function that depends on the metric of the configation space.

According to a result of [10] (see also [16]), if an integral of motion of the mechanical system exists in the form of a polynomial of the fourth degree in velocities, this integral can be reduced while preserving the form of the Lagrangian (1) to the form

$$
I=\xi^{\prime 4}+P \xi^{\prime 2}+Q \xi^{\prime} \eta^{\prime}+R=I_{0}(\text { const })
$$

where $R$ is given by the quadrature

$$
R=-\int Q \frac{\partial U}{\partial \xi} d \eta-\int\left[2 P \frac{\partial U}{\partial \xi}+Q \frac{\partial U}{\partial \eta}+2 U \frac{\partial Q}{\partial \eta}\right]_{0} d \xi
$$

in which []$_{0}$ means that the expression in the bracket is computed for $\eta$ taking an arbitrary constant value $\eta_{0}$ (say), and the other three functions involved are expressed as

$$
P=\frac{\partial^{2} F}{\partial \xi^{2}}, Q=-\frac{\partial^{2} F}{\partial \xi \partial \eta}, U=-\frac{1}{4}\left(\frac{\partial^{2} F}{\partial \xi^{2}}+\frac{\partial^{2} F}{\partial \eta^{2}}\right)
$$

in terms of a single generating function $F$ which satisfies the nonlinear partial differential equation

$$
\begin{aligned}
e= & \frac{\partial^{2} F}{\partial \xi \partial \eta}\left(\frac{\partial^{4} F}{\partial \xi^{4}}-\frac{\partial^{4} F}{\partial \eta^{4}}\right)+3\left(\frac{\partial^{3} F}{\partial \xi^{3}} \frac{\partial^{3} F}{\partial \xi^{2} \partial \eta}-\frac{\partial^{3} F}{\partial \eta^{3}} \frac{\partial^{3} F}{\partial \eta^{2} \partial \xi}\right) \\
& +2\left(\frac{\partial^{2} F}{\partial \xi^{2}} \frac{\partial^{4} F}{\partial \xi^{3} \partial \eta}-\frac{\partial^{2} F}{\partial \eta^{2}} \frac{\partial^{4} F}{\partial \eta^{3} \partial \xi}\right) \\
= & 0
\end{aligned}
$$

The set of solutions of this equation generates all systems of the type (11) having an integral of the form (4) on the zero level of their energy integral. Affecting all possible conformal mappings of the complex $\zeta=\xi+i \eta$ plane followed by a general point transformation to the generalized coordinates $q_{1}, q_{2}$ with a suitable change of the time variable we obtain all systems of the general form on $2 \mathrm{D}$ Riemannian (or pseudo-Riemannian) manifolds, having a quartic integral on the zero level of their energy integral. 


\subsection{Certain forms of solutions of the resolving equation}

The fourth-order PDE (7) is homogeneous nonlinear of the second degree. It is not known whether this equation is integrable, in the sense that some procedure can be pointed out to construct all its solutions, nor it can be reduced to an equation of lower order. The best one can do is to try to construct solutions involving as much arbitrary parameters as possible.

1. Equation (7) admits a solution of the form

$$
F=A \Phi(\xi) \Psi(\eta)
$$

where $A$ is a constant and both $\Phi$ and $\Psi$ satisfy the same equation

$$
\Phi^{(4)}(\xi)+5 \frac{\Phi^{\prime \prime}(\xi) \Phi^{\prime \prime \prime}(\xi)}{\Phi^{\prime}(\xi)}-6 a_{4} \Phi(\xi)=0
$$

in which $a_{4}$ is a separation constant. Comparing with the results of [16 we conclude that the original isometric variables $\xi, \eta$ are not practically suitable coordinates for the description of the solution, and that a symmetric separation solution (8) can be more conveniently expressed in the form

$$
F=A p q
$$

in terms of the pair of variables $p, q$ related to $\xi, \eta$ by the relations

$$
\begin{aligned}
& \xi=\int^{p} \frac{d p}{\sqrt[4]{a_{4} p^{4}+a_{2} p^{2}+a_{1} p+a_{0}}} \\
& \eta=\int^{q} \frac{d q}{\sqrt[4]{a_{4} q^{4}+b_{2} q^{2}+b_{1} q+b_{0}}}
\end{aligned}
$$

The three constants $a_{2}, a_{1}, a_{0}$ (and $b_{2}, b_{1}, b_{0}$ ) are integration constants of the fourth-order equation (9) for $\Phi$ (and $\Psi$ ). The fourth constant is eliminated by a shift of the variable $p(q)$.

2. A quite rich system, first announced in [16] as the master system, can be derived from the solution

$$
F=F_{0}(p)+G_{0}(q)+\nu p q
$$

in which

$$
\begin{aligned}
F_{0} & =\int^{p} \frac{d p}{\sqrt[4]{a_{4} p^{4}+a_{3} p^{3}+a_{2} p^{2}+a_{1} p+a_{0}}} \int^{p} \frac{\left(4 C_{0}+4 C_{1} p+4 A p^{2}+\frac{1}{4} b_{3} p^{3}\right) d p}{\left(a_{4} p^{4}+a_{3} p^{3}+a_{2} p^{2}+a_{1} p+a_{0}\right)^{3 / 4}} \\
G_{0} & =\int^{q} \frac{d q}{\sqrt[4]{a_{4} q^{4}+b_{3} q^{3}+b_{2} q^{2}+b_{1} q+b_{0}}} \int^{q} \frac{\left(4 D_{0}+4 D_{1} q+4 A q^{2}+\frac{1}{4} a_{3} q^{3}\right) d q}{\left(a_{4} q^{4}+b_{3} q^{3}+b_{2} q^{2}+b_{1} q+b_{0}\right)^{3 / 4}}
\end{aligned}
$$


and the relations

$$
\begin{aligned}
\xi & =\int^{p} \frac{d p}{\sqrt[4]{a_{4} p^{4}+a_{3} p^{3}+a_{2} p^{2}+a_{1} p+a_{0}}} \\
\eta & =\int^{q} \frac{d q}{\sqrt[4]{a_{4} q^{4}+b_{3} q^{3}+b_{2} q^{2}+b_{1} q+b_{0}}}
\end{aligned}
$$

generalize (10) by a shift of the two variables $p, q$. This system has the Lagrangian

$$
\begin{aligned}
L= & \frac{1}{2}\left[\frac{\dot{p}^{2}}{\sqrt{a_{4} p^{4}+a_{3} p^{3}+a_{2} p^{2}+a_{1} p+a_{0}}}+\frac{\dot{q}^{2}}{\sqrt{a_{4} q^{4}+b_{3} q^{3}+b_{2} q^{2}+b_{1} q+b_{0}}}\right] \\
& -\left[\frac{\nu b_{3} p^{3}+A p^{2}+C_{1} p+C_{0}}{\sqrt{a_{4} p^{4}+a_{3} p^{3}+a_{2} p^{2}+a_{1} p+a_{0}}}+\frac{\nu a_{3} q^{3}+A q^{2}+D_{1} q+D_{0}}{\sqrt{a_{4} q^{4}+b_{3} q^{3}+b_{2} q^{2}+b_{1} q+b_{0}}}\right] \\
& -\nu\left[\frac{q\left(4 a_{4} p^{3}+3 a_{3} p^{2}+2 a_{2} p+a_{1}\right)}{\sqrt{a_{4} p^{4}+a_{3} p^{3}+a_{2} p^{2}+a_{1} p+a_{0}}}+\frac{p\left(4 a_{4} q^{3}+3 b_{3} q^{2}+2 b_{2} q+b_{1}\right.}{\sqrt{a_{4} q^{4}+b_{3} q^{3}+b_{2} q^{2}+b_{1} q+b_{0}}}\right]
\end{aligned}
$$

which involves 15 free parameters $a_{0}, a_{1}, a_{2}, a_{3}, a_{4}, b_{0}, b_{1}, b_{2}, b_{3}, A, C_{0}, C_{1}, D_{0}, D_{1}, \nu$ and the quartic integral valid only on the zero level of its energy. This integral was provided in [16]. It turned out that the integral can be reduced to somewhat simpler form

$$
\begin{aligned}
I= & \frac{\left\{\dot{p}^{2} / 2+\nu q\left(4 a_{4} p^{3}+3 a_{3} p^{2}+2 a_{2} p+a_{1}\right)+\nu b_{3} p^{3}+C_{1} p+C_{0}\right\}^{2}}{a_{4} p^{4}+a_{3} p^{3}+a_{2} p^{2}+a_{1} p+a_{0}}-4 \nu \dot{p} \dot{q} \\
& -8 \nu^{2} \sqrt{a_{4} p^{4}+a_{3} p^{3}+a_{2} p^{2}+a_{1} p+a_{0}} \sqrt{a_{4} q^{4}+b_{3} q^{3}+b_{2} q^{2}+b_{1} q+b_{0}} \\
& \left.-4 \nu\left\{\nu\left[b_{2} p^{2}+a_{2} q^{2}+3 p q\left(2 a_{4} p q+b_{3} p+a_{3} q\right)\right]+2 A p q+D_{1} p+C_{1} q\right\} 5\right)
\end{aligned}
$$

\section{Real dynamics:}

\subsection{Classes of time-parametrized geodesics.}

To construct a system that would be a priori integrable on all levels of energy, we should receive a function $U$ from (6) that has the structure (3), i.e. involving in a linear way an arbitrary parameter $h$. In that case The generating function should have the form $F=h F_{0}+F_{1}$, where $F_{0}, F_{1}$ are functions not depending on $h$. This is usually expected when solving linear PDEs. However, we shall rely on solutions of the nonlinear PDE (7) having this property of being a linear superposition of solutions. It is obvious from (7) that the function $F$ can always be reduced to the forms $\lambda F_{0}$ ( $\lambda$ arbitrary constant), but in practice we seek $F$ in the form of a linear superposition of such expressions, so that

$$
F=\sum_{i=1}^{k} \lambda_{i} F_{i}
$$


where $\lambda_{i}, i=1, \ldots, k$ are arbitrary parameters and $\left\{F_{i}\right\}$ do not depend on any one of the $\lambda_{i}$ s. This choice will lead to

$$
U=\sum_{i=1}^{k} \lambda_{i} U_{i}, U_{i}=-\frac{1}{4}\left(\frac{\partial^{2} F_{i}}{\partial \xi^{2}}+\frac{\partial^{2} F_{i}}{\partial \eta^{2}}\right)
$$

and $\left\{U_{i}\right\}$ do not depend on any one of the $\lambda_{i}$ s. Each $\lambda_{i}$ can be regarded as the energy parameter of the real mechanical system before reduction to the form (11), and hence we call them energy-like parameters. However, for a much general result we formulate the following

\section{Theorem 1}

Let the system with the (reduced) Lagrangian

$$
L=\frac{1}{2}\left[\xi^{\prime 2}+\eta^{\prime 2}\right]+\sum_{i=1}^{k} \lambda_{i} U_{i}
$$

in which ()$^{\prime}=\frac{d}{d \tau}$ and $\left\{U_{i}\right\}$ do not depend on any one of the $\lambda_{j}$, admit on its zero-energy level an integral

$$
I=I\left(\xi, \eta, \xi^{\prime}, \eta^{\prime}, \lambda_{1}, \cdots, \lambda_{k}\right)
$$

then the Lagrangian

$$
L^{*}=\frac{1}{2}\left(\sum_{i=1}^{k} \alpha_{i} U_{i}\right)\left[\dot{\xi}^{2}+\dot{\eta}^{2}\right]+\frac{\sum_{i=1}^{k} \beta_{i} U_{i}}{\sum_{i=1}^{k} \alpha_{i} U_{i}}
$$

in which $\left(\dot{)}=\frac{d}{d t}\right.$ and $t$ is the natural time, admits an unconditional integral (valid for arbitrary initial conditions) of the form

$$
I^{*}=I\left(\xi, \eta, \Lambda \dot{\xi}, \Lambda \dot{\eta}, \alpha_{1} h+\beta_{1}, \cdots, \alpha_{k} h+\beta_{k}\right)
$$

where $\alpha_{1}, \ldots, \alpha_{k}, \beta_{1}, \ldots, \beta_{k}$ are arbitrary parameters, $\Lambda=\sum_{i=1}^{k} \alpha_{i} U_{i}$ and $h$ is the energy integral for (20). The energy parameter $h$ can be either substituted by its value taken on an arbitrary motion of the system(20) or replaced by its functional form in the state space

$$
h=\frac{1}{2}\left(\sum_{i=1}^{k} \alpha_{i} U_{i}\right)\left[\dot{\xi}^{2}+\dot{\eta}^{2}\right]-\frac{\sum_{i=1}^{k} \beta_{i} U_{i}}{\sum_{i=1}^{k} \alpha_{i} U_{i}}
$$

\section{Proof}


Introduce new arbitrary parameters $\left\{\alpha_{i}, \beta_{i}\right\}$ by the substitution $\lambda_{i}=\alpha_{i} h+$ $\beta_{i}, i=1, \ldots, k$. The reduced force function in (18) splits into the two expressions $\sum_{i=1}^{k} \beta_{i} U_{i}+h \sum_{i=1}^{k} \alpha_{i} U_{i}$. Now, we make the change of the independent variable $\tau$ to the original (natural) time variable according to the relation

$$
d \tau=\frac{d t}{\Lambda}, \Lambda=\sum_{i=1}^{k} \alpha_{i} U_{i}
$$

It is easy to see that those substitutions lead to the Lagrangian

$$
L_{1}=L^{*}+h
$$

The additive parameter $h$ in $L_{1}$ is insignificant and discarding it reduces $L_{1}$ to $L^{*}$. The zero level of the energy integral for $L_{1}$ is the same as the $h$-level for $L^{*}$.

Notes:

1. Although we are concerned here with systems with a quartic integral, in this theorem the integral (19) is a general function of its arguments and not necessarily quartic nor even a polynomial in velocities.

2. According to Maupertuis principle (see e.g. 20]), it should be noted that the whole $2 k$ - parameter family of mechanical systems (20) on their level $h$ of energy share one and the same $k$-parameter geodesic flow (or the same $k$ - parameter family of unparametrized trajectories) on the manifold with metric

$$
d s^{2}=2\left(\sum_{i=1}^{k} \lambda_{i} U_{i}\right)\left[d \xi^{2}+d \eta^{2}\right]
$$

3. The form (18) in the independent variable $\tau$ can be regarded as the simplest form of the $2 k$ - parameter family of mechanical systems. Let $(\xi(\tau), \eta(\tau))$ be the general solution of (18). The general solution of (20) can be expressed in terms of the natural time $t$ by first integrating the relation (22) to get

$$
t=\int \Lambda d \tau=\int \sum_{i=1}^{k} \alpha_{i} U_{i}(\xi(\tau), \eta(\tau)) d \tau
$$

and solving for $\tau$ we obtain $\tau=\tau(t)$, so that the solution of (20) is $(\xi(\tau(t)), \eta(\tau(t)))$. The form of the function $\tau(t)$ for each choice of the set of parameters $\left\{\alpha_{i}\right\}$ determines the way in which the mechanical system (20), which lives on the -generally speaking- non-Euclidean manifold with metric

$$
d s^{2}=2\left(\sum_{i=1}^{k} \alpha_{i} U_{i}\right)\left[d \xi^{2}+d \eta^{2}\right]
$$

describes its trajectory in the plane of the isometric variables $\xi, \eta$. 


\section{A new solution of the resolving equation}

In the present section we try a deformation of the master system to accomodate on more term of the product type, probably at the expense of enforcing certain restrictions on some of the parameters figuring in (12). In fact, we assume F in the form

$$
F=F_{0}+K_{1} p q+K_{2} p^{2} q^{2}
$$

Inserting the expression (25) in equation (7) and using the relations (13), we obtain after some manipulations a polynomial expression in the two variables $p, q$ that must vanish identically. Those equations have been solved under the condition that $a_{4} \neq 0$, so that the two polynomials that occur under the fourth degree root signs are of the fourth degree. This resulted in only one new case, which differs from the master system of [16]. This case will be considered in detail in the next subsections.

\subsection{The generic restricted case}

We write the Lagrangian and the comlementary integral for this case after some transformation to more symmetric form that does not affect the generality of the system (arbitrary shifts of the two variables will retain the raw case):

$$
\begin{gathered}
L=\frac{1}{2}\left[\frac{\dot{u}^{2}}{\sqrt{a u^{4}+k_{1} u^{2}+k_{0}}}+\frac{\dot{v}^{2}}{\sqrt{a v^{4}+m_{1} v^{2}+m_{0}}}\right]+U \\
U=-\frac{N\left[\left(4 a v^{2}+m_{1}\right)\left(4 a u^{4}+3 k_{1} u^{2}+2 k_{0}\right)\right]+\nu u v\left(2 a u^{2}+k_{1}\right)+K u^{2}+D}{2 \sqrt{a u^{4}+k_{1} u^{2}+k_{0}}} \\
-\frac{N\left[\left(4 a u^{2}+k_{1}\right)\left(4 a v^{4}+3 m_{1} v^{2}+2 m_{0}\right)\right]+\nu u v\left(2 a v^{2}+m_{1}\right)+K v^{2}+E}{2 \sqrt{a v^{4}+m_{1} v^{2}+m_{0}}}(27) \\
I=\quad \frac{\left\{u^{2}+N\left(4 a v^{2}+m_{1}\right)\left(4 a u^{4}+3 k_{1} u^{2}+2 k_{0}\right)+\nu u v\left(2 a u^{2}+k_{1}\right)+K u^{2}+D\right\}^{2}}{\left(a u^{4}+k_{1} u^{2}+k_{0}\right)} \\
-4(8 N a u v+\nu) u^{\prime}-2(8 N a u v+\nu)^{2} \sqrt{a u^{4}+k_{1} u^{2}+k_{0}} \sqrt{a v^{4}+m_{1} v^{2}+m_{0}} \\
-32 a N^{2}\left[u^{2}\left(2 a u^{2}+k_{1}\right)\left(6 a v^{4}+3 m_{1} v^{2}+m_{0}\right)+k_{0} v^{2}\left(2 a v^{2}+m_{1}\right)\right] \\
-16 N a\left(E u^{2}+D v^{2}+2 K u^{2} v^{2}\right) \\
-4 N \nu u v\left[8 a\left(m_{1} u^{2}+k_{1} v^{2}+3 a u^{2} v^{2}\right)+3 k_{1} m_{1}\right] \\
-\nu^{2}\left(m_{1} u^{2}+k_{1} v^{2}+6 a u^{2} v^{2}\right)-4 \nu K u v
\end{gathered}
$$

in which the prime represents derivative with respect to the independent variable $\tau$ (ficticious time). Note that when the two parameters $N$ and $\nu$ vanish the Lagrangian (26) degenerates into a separable one and the integral (28) into the square of a quadratic integral. 
The system with the Lagrangian (26) admits the integral (28) only on the zero-energy level of this system

$$
\frac{1}{2}\left[\frac{\hat{u}^{2}}{\sqrt{a u^{4}+k_{1} u^{2}+k_{0}}}+\frac{\hat{v}^{2}}{\sqrt{a v^{4}+m_{1} v^{2}+m_{0}}}\right]-U=0
$$

This system involves 10 parameters $a, k_{0}, k_{1}, m_{0}, m_{1}, D, E, K, \nu, N$, of which the first five enter in both the kinetic energy and potential terms of the Lagrangian and the last five ones enter only in the potential terms and, moreover, they enter only linearly. The last four parameters constitute a set of energy-like parameters (see e.g. [16]). They are essential in building the unrestricted integrable system. Comparing (26) to its counterpart in the "master" system [16, we find that (26) involves only one new parameter $N$, which is not present in the master system. When $N$ is set equal to zero (26) turns out to be a special case of the master system resulting from one restriction on the coefficients of each of the two fourth-degree polynomials entering under the root sign and two restrictions on the energy -like parameters (namely, the vanishing of the parameters $C_{1}, D_{1}$ of [16]).

\subsection{Dynamics- The unrestricted generalization}

We now proceed to use those parameters to construct a general integrable system valid on arbitrary energy level out of the restricted one. Introducing new parameters by the relations

$$
\begin{aligned}
D & =h_{1}-h \alpha_{1}, E=h_{2}-h \alpha_{2}, \\
K & =h_{3}-h \alpha_{3}, \nu=h_{4}-h \alpha_{4}, \\
N & =h_{5}-h \alpha_{5}
\end{aligned}
$$

and performing the change of independent variable to the actual-time parametrization by using the relation

$$
d \tau=\frac{d t}{\Lambda}
$$

where

$$
\begin{aligned}
\Lambda= & \frac{\alpha_{1}+\alpha_{3} u^{2}+\alpha_{4} u v\left(2 a u^{2}+k_{1}\right)+\alpha_{5}\left[m_{1} u^{4}+v^{2}\left(4 a u^{4}+3 k_{1} u^{2}+2 k_{0}\right)\right]}{\sqrt{a u^{4}+k_{1} u^{2}+k_{0}}} \\
& +\frac{\alpha_{2}+\alpha_{3} v^{2}+\alpha_{4} u v\left(2 a v^{2}+m_{1}\right)+\alpha_{5}\left[k_{1} v^{4}+u^{2}\left(4 a v^{4}+3 m_{1} v^{2}+2 m_{8}\right)\right]}{\sqrt{a v^{4}+m_{1} v^{2}+m_{0}}}
\end{aligned}
$$

we arrive at the new Lagrangian

$$
L=\frac{1}{2} \Lambda\left[\frac{\dot{u}^{2}}{\sqrt{a u^{4}+k_{1} u^{2}+k_{0}}}+\frac{\dot{v}^{2}}{\sqrt{a v^{4}+m_{1} v^{2}+m_{0}}}\right]-V+h
$$




$$
\begin{aligned}
V= & \frac{1}{\Lambda}\left\{\frac{h_{1}+h_{3} u^{2}+h_{4} u v\left(2 a u^{2}+k_{1}\right)+h_{5}\left[m_{1} u^{4}+v^{2}\left(4 a u^{4}+3 k_{1} u^{2}+2 k_{0}\right)\right]}{\sqrt{a u^{4}+k_{1} u^{2}+k_{0}}}\right. \\
& +\frac{h_{2}+h_{3} v^{2}+h_{4} u v\left(2 a v^{2}+m_{1}\right)+h_{5}\left[k_{1} v^{4}+u^{2}\left(4 a v^{4}+3 m_{1} v^{2}+2 m_{0}\right)\right.}{\sqrt{a v^{4}+m_{1} v^{2}+m_{0}}}
\end{aligned}
$$

which admits on an arbitrary energy level $h$ the integral resulting from (28) by the substitutions (29) and (30), i.e. $\dot{u} \rightarrow \Lambda \dot{u}, \dot{v} \rightarrow \Lambda \dot{v}$. The integral will depend on the parameters occuring in the Lagrangian and also on the energy constant $h$. The last constant may be substituted by its expression in terms of the coordinates and velocities to get the final form free of the energy restriction. The resulting system depends on 16 parameters $a, k_{0}, k_{1}, m_{0}, m_{1}, \alpha_{1}, \alpha_{2}, \alpha_{3}, \alpha_{4}, \alpha_{5}, h_{1}, h_{2}, h_{3}, h_{4}, h_{5}$ and $h$, of which the first nine enter in both the kinetic energy (the metric of the configuration space) and potential terms of the Lagrangian but the last five ones enter only in the potential.

\subsection{Special cases}

\subsubsection{Generalization of the cases of Bozis and Wojciechowski}

Let $a=k_{0}=m_{0}=1, k_{1}=m_{1}=-2$. Under the coordinate transformation $p=\sin y, q=\sin x$ the Lagrangian (32) takes the form

$$
\begin{aligned}
L= & \frac{1}{2}\left[\alpha+\beta \sin x \sin y+\gamma\left(2 \cos ^{2} x \cos ^{2} y-\cos ^{2} x-\cos ^{2} y\right)+\frac{\delta_{1}}{\cos ^{2} x}+\frac{\delta_{2}}{\cos ^{2} y}\right]\left(\dot{x}^{2}+\dot{y}^{2}\right) \\
& -\frac{a+b \sin x \sin y+c\left(2 \cos ^{2} x \cos ^{2} y-\cos ^{2} x-\cos ^{2} y\right)+\frac{d_{1}}{\cos ^{2} x}+\frac{d_{2}}{\cos ^{2} y}}{\alpha+\beta \sin x \sin y+\gamma\left(2 \cos ^{2} x \cos ^{2} y-\cos ^{2} x-\cos ^{2} y\right)+\frac{\delta_{1}}{\cos ^{2} x}+\frac{\delta_{2}}{\cos ^{2} y}}
\end{aligned}
$$

When $\beta=\gamma=\delta_{1}=\delta_{2}=0$ we have, after ignoring an insignificant additive constant

$L=\frac{1}{2}\left(\dot{x}^{2}+\dot{y}^{2}\right)-\left[b \sin x \sin y+c\left(2 \cos ^{2} x \cos ^{2} y-\cos ^{2} x-\cos ^{2} y\right)+\frac{d_{1}}{\cos ^{2} x}+\frac{d_{2}}{\cos ^{2} y}\right]$

This system is new. It describes plane motion of a particle in a 4-parameter potential. The complementary integral of this system can be written as

$$
\begin{aligned}
I= & \left(\dot{x}^{2}+\frac{2 d_{1}}{\cos ^{2} x}\right)\left(\dot{y}^{2}+\frac{2 d_{2}}{\cos ^{2} y}\right)-2 \cos x \cos y(b+2 c \sin x \sin y) \dot{x} \dot{y} \\
& +\cos ^{2} x \cos ^{2} y(b+2 c \sin x \sin y)^{2}+4 c\left(d_{1} \cos ^{2} y+d_{2} \cos ^{2} x\right)
\end{aligned}
$$

When $c=0$ this case reduces to a special version of that found by Bozis [21] and when $c=b=0$ the system becomes separable and the integral degenerates into the product of two quadratic integrals. A slight variation of the parameters in (34) to be $k_{1}=m_{1}=2$ changes trigonometric functions to hyperbolic (or 
exponential) functions, and thus giving a new system like the type of [23]. The analog of (35) gives a particle in the potential

$V=b \sinh x \sinh y+c\left(2 \cosh ^{2} x \cosh ^{2} y-\cosh ^{2} x-\cosh ^{2} y\right)+\frac{d_{1}}{\cosh ^{2} x}+\frac{d_{2}}{\cosh ^{2} y}$

with the corresponding integral

$$
\begin{aligned}
I= & \left(\dot{x}^{2}+\frac{2 d_{1}}{\cosh ^{2} x}\right)\left(\dot{y}^{2}+\frac{2 d_{2}}{\cosh ^{2} y}\right)+2 \cosh x \cosh y(-b+2 c \sinh x \sinh y) \dot{x} \dot{y} \\
& +\cosh ^{2} x \cosh ^{2} y(b-2 c \sinh x \sinh y)^{2}+4 c\left(d_{1} \cosh ^{2} y+d_{2} \cosh ^{2} x\right)(38)
\end{aligned}
$$

In a similar way, one can obtain a mix of the two types by taking $k_{1}=-m_{1}=2$.

\subsubsection{Systems of the Toda type}

If in (32) we set $a=1, k_{0}=k_{1}=m_{0}=m_{1}=0$, the Lagrangian takes the form

$$
\begin{aligned}
L= & \frac{1}{2}\left(\alpha_{0}+\alpha e^{-2 x}+\beta e^{-2 y}+\gamma e^{x+y}+\delta e^{2(x+y)}\right)\left(\dot{x}^{2}+\dot{y}^{2}\right) \\
& -\frac{h_{0}+a e^{-2 x}+b e^{-2 y}+c e^{x+y}+d e^{2(x+y)}}{\alpha_{0}+\alpha e^{-2 x}+\beta e^{-2 y}+\gamma e^{x+y}+\delta e^{2(x+y)}}
\end{aligned}
$$

and the integral may be written, after using the energy integral to eliminate $h$, as

$$
\begin{aligned}
I= & \lambda^{4} \dot{x}^{2} \dot{y}^{2}+2 \lambda^{2}\left[b e^{-2 y} \dot{x}^{2}+a e^{-2 x} \dot{y}^{2}+\left(c e^{x+y}+d e^{2 x+2 y}\right) \dot{x} \dot{y}\right] \\
& +e^{2 x+2 y}\left(c+d e^{x+y}\right)^{2}+2 d\left(b e^{2 x}+a e^{2 y}\right)+4 a b e^{-2 x-2 y}
\end{aligned}
$$

where $\lambda=\alpha_{0}+\alpha e^{-2 x}+\beta e^{-2 y}+\gamma e^{x+y}+\delta e^{2(x+y)}$.

A special case of the Toda type is

$$
L=\frac{1}{2}\left(\dot{x}^{2}+\dot{y}^{2}\right)-\left(a e^{-2 x}+b e^{-2 y}+c e^{x+y}+d e^{2(x+y)}\right)
$$

In two more special cases the configuration space degenerates into a plane. Their lagrangians can be written in polar coordinates as done in [16]:

$$
\begin{aligned}
& L_{1}=\frac{1}{2}\left(\dot{r}^{2}+r^{2} \dot{\theta}^{2}\right)-\left(A r^{2}+\frac{B}{r^{2}}+\frac{C e^{2 \theta}+D e^{-2 \theta}}{r^{4}}\right) \\
& L_{2}=\frac{1}{2}\left(\dot{r}^{2}+r^{2} \dot{\theta}^{2}\right)-\left(\frac{A+B e^{-2 \theta}}{r^{2}}+\frac{C e^{\theta}}{r^{3}}+\frac{D e^{2 \theta}}{r^{4}}\right)
\end{aligned}
$$

The last three cases of motion in the plane seem to be new, but their potentials are not periodic in $\theta$. 


\subsection{Application to rigid body dynamics}

We now consider the general problem of motion of a rigid body about a fixed point under the action of a combination of conservative axisymmetric potential forces. The equations of motion for this problem can be written in the EulerPoisson form:

$$
\begin{aligned}
A \dot{p}+(C-B) q r & =\gamma_{2} \frac{\partial V}{\partial \gamma_{3}}-\gamma_{3} \frac{\partial V}{\partial \gamma_{2}}, \\
B \dot{q}+(A-C) p r & =\gamma_{3} \frac{\partial V}{\partial \gamma_{1}}-\gamma_{1} \frac{\partial V}{\partial \gamma_{3}}, \\
C \dot{r}+(B-A) p q & =\gamma_{1} \frac{\partial V}{\partial \gamma_{2}}-\gamma_{2} \frac{\partial V}{\partial \gamma_{1}}, \\
\dot{\gamma}_{1}+q \gamma_{3}-r \gamma_{2}=0, \dot{\gamma}_{2}+r \gamma_{1} & -p \gamma_{3}=0, \dot{\gamma}_{3}+p \gamma_{2}-q \gamma_{1}=0,
\end{aligned}
$$

where $A, B, C$ are the principal moments of inertia, $p, q, r$ are the components of the angular velocity of the body and $\gamma_{1}, \gamma_{2}, \gamma_{3}$ are the components of the unit vector $\gamma$ fixed in space in the direction of the axis of symmetry of the force fields applied to the body, all being referred to the principal axes of inertia at the fixed point.

The system (44) admits three integrals:

$$
\begin{aligned}
I_{1} & =\frac{1}{2}\left(A p^{2}+B q^{2}+C r^{2}\right)+V \\
I_{2} & =A p \gamma_{1}+B q \gamma_{2}+C r \gamma_{3} \\
I_{3} & =\gamma_{1}^{2}+\gamma_{2}^{2}+\gamma_{3}^{2}=1
\end{aligned}
$$

Equations (44) admit an equivalent representation in the Lagrangian form (see e.g. [16]), which we write here for a dynamically symmetric body, for which $B=A$. As generalized coordinates we use the Eulerian angles: $\psi$ the angle of precession around the axis of symmetry of the field, $\theta$ the angle of nutation and $\varphi$ the angle of proper rotation (about the axis of symmetry of the body). The components of the vector $\gamma$ can be expressed as

$$
\gamma_{1}=\sin \theta \sin \varphi, \gamma_{2}=\sin \theta \cos \varphi, \gamma_{3}=\cos \theta
$$

After ignoring the cyclic variable $\psi$ in the sense of Routh on the zero level of the cyclic integral $I_{2}=0$, the Routhian of this mechanical system expressed in the other two angles $\theta$ and $\varphi$ has the form

$$
R=\frac{1}{2} A\left[\dot{\theta}^{2}+\frac{C \sin ^{2} \theta \dot{\varphi}^{2}}{A-(A-C) \cos ^{2} \theta}\right]-V
$$

Comparing the structure of this Routhian function to that of the Lagrangian (32) and recalling the procedure followed in a similar situation in [16], we get 
convinced that they become identical only in the case of Kovalevskaya configuration $A=B=2 C$.

In fact, setting $a=1, k_{1}=1, k_{0}=0, m_{1}=2, m_{0}=1, \alpha_{1}=\alpha_{4}=\alpha_{5}=$ $0, \alpha_{2}=\alpha_{3}$, and affecting the substitution $u=\frac{\cos ^{2} \theta}{2 \sin \theta}, v=\cos \varphi$ and renaming the remaining parameters, we get the Lagrangian

$$
\begin{aligned}
L & =\frac{1}{2}\left[\dot{\theta}^{2}+\frac{\sin ^{2} \theta}{1+\sin ^{2} \theta} \dot{\varphi}^{2}\right]-V, \\
V & =2 C\left[a \sin \theta \sin \varphi+b \sin ^{2} \theta \cos (2 \varphi)+\frac{\lambda}{\cos ^{2} \theta}+\mu \frac{1+\sin ^{2} \theta}{\sin ^{2} \theta \cos ^{2} \varphi}\right]
\end{aligned}
$$

and the integral

$$
\begin{aligned}
I= & \frac{\sin ^{6} \theta \dot{\varphi}^{4}}{\left(1+\sin ^{2} \theta\right)^{4}} \\
& +\frac{\sin ^{2} \theta \dot{\varphi}^{2}}{\left(1+\sin ^{2} \theta\right)^{2}}\left[2 a \sin \theta \sin \varphi+b\left(2-3 \cos ^{2} \theta\right)+\frac{2 \lambda \sin ^{2} \theta}{\cos ^{2} \theta}+\frac{4 \mu}{\cos ^{2} \varphi}+\sin ^{2} \theta \dot{\theta}^{2}\right] \\
& +\frac{2 \dot{\theta} \dot{\varphi} \sin \theta \cos \theta \cos \varphi}{\left(1+\sin ^{2} \theta\right)}\left(a \sin \theta+2 b \cos ^{2} \theta \sin \varphi\right)+\dot{\theta}^{2}\left[b \cos 2 \varphi+\frac{2 \mu}{\cos ^{2} \varphi}\right] \\
& +\frac{4 \mu^{2}}{\sin ^{2} \theta \cos ^{4} \varphi}+\mu\left[\frac{4 \lambda}{\cos ^{2} \theta \cos ^{2} \varphi}+\frac{4 a \sin \varphi}{\sin \theta \cos ^{2} \varphi}+2 b\left(2 \sin ^{2} \theta+\frac{1-3 \sin ^{2} \theta}{\sin ^{2} \theta \cos ^{2} \varphi}\right)\right] \\
& +\left(\frac{1}{2} a^{2}-2 a b \sin \theta \sin \varphi\right)\left(1-2 \sin ^{2} \theta \cos ^{2} \varphi\right)+\frac{1}{2} b^{2} \sin ^{2} \theta\left[\sin ^{2} \theta(\cos 4 \varphi-1)+4\right] \\
& +2 \lambda b \tan ^{2} \theta \cos 2 \varphi
\end{aligned}
$$

To facilitate comparison with other results, we now express the last integrable case of rigid body dynamics in the Euler-Poisson variables, i.e. as a solution of the system (44), in the next

\section{Theorem 2:}

For a rigid body with moments of inertia satisfying $A=B=2 C$ and for the potential

$$
V=2 C\left[a \gamma_{1}+b\left(\gamma_{1}^{2}-\gamma_{2}^{2}\right)+\frac{\lambda}{\gamma_{3}^{2}}+\delta \frac{2-\gamma_{3}^{2}}{\gamma_{2}^{2}}\right]
$$

equations (44) are integrable on the level $I_{2}=0$. The complementary integral has the form

$$
\begin{aligned}
I= & \left(p^{2}-q^{2}-a \gamma_{1}+b \gamma_{3}^{2}-\frac{\lambda\left(\gamma_{1}^{2}-\gamma_{2}^{2}\right)}{\gamma_{3}^{2}}\right)^{2}+\left(2 p q-a \gamma_{2}-\frac{2 \lambda \gamma_{1} \gamma_{2}}{\gamma_{3}^{2}}\right)^{2} \\
& +\frac{\delta}{\gamma_{2}^{2}}\left[2\left(p^{2}+q^{2}\right) \gamma_{3}^{2}-2 a \gamma_{1} \gamma_{3}^{2}-2 \lambda \gamma_{1}^{2}+2 b+\frac{\delta \gamma_{3}^{4}}{\gamma_{2}^{2}}\right]
\end{aligned}
$$


This case is new. For comparison we provide a table of presently known integrable potentials related to the type (52), which admit a quartic integral under the condition $A=B=2 C$ :

Author- year Potential

Kovalevskaya [1]1889 $V_{1}=a_{1} \gamma_{1}+a_{2} \gamma_{2}$

Chaplygin [24] $1903 \quad V_{2}=b_{1}\left(\gamma_{1}^{2}-\gamma_{2}^{2}\right)+b_{2} \gamma_{1} \gamma_{2}$

Goriatchev [25] $1916 \quad V_{3}=a \gamma_{1}+a_{2} \gamma_{2}+b\left(\gamma_{1}^{2}-\gamma_{2}^{2}\right)+b_{1} \gamma_{1} \gamma_{2}+\frac{\lambda}{\gamma_{3}^{2}}$

Yehia [16] 2006

$$
\begin{aligned}
& V_{4}=b\left(\gamma_{1}^{2}-\gamma_{2}^{2}\right)+\frac{\lambda}{\gamma_{3}^{2}}+\rho\left(\frac{1}{\gamma_{3}^{4}}-\frac{1}{\gamma_{3}^{6}}\right)+\left(2-\gamma_{3}^{2}\right)\left(\frac{\nu}{\gamma_{1}^{2}}+\frac{\delta}{\gamma_{2}^{2}}\right) \\
& V_{5}=a \gamma_{1}+\frac{\lambda}{\gamma_{3}^{2}}+\frac{\varepsilon}{\sqrt{\gamma_{1}^{2}+\gamma_{2}^{2}}}+\frac{\left(2-\gamma_{3}^{2}\right)}{\gamma_{2}^{2}}\left[\delta+\mu \frac{\gamma_{1}}{\sqrt{\gamma_{1}^{2}+\gamma_{2}^{2}}}\right]
\end{aligned}
$$

The potential (52) involves a collection of parameters: $a$ of the Kovalevskaya type, $b$ of the Chaplygin type, the Goriatchev parameter $\lambda$ and the parameter $\delta$ figuring in both new cases announced in our work [16, but the combination (52) is new.

\section{References}

[1] Kowalevski S.V., Sur le probleme de la rotation d'un corps solide autour d'un point fixe. Acta Math. 12, 177-232 (1889).

[2] Hietarinta J., Direct Methods for the search of the second Invariant, , Phys. Rep., (Review Section of Physics Letters), Vol. 147, No. 2 (1987), pp. 87154.

[3] Karlovini M., Pucacco G, Rosquist K and Samuelsson L., A unified treatment of quartic invarients at fixed and arbitrary integral, J. of Mathematical physics, Vol. 43, No. 8, , (2002), pp. 4041-4059.

[4] Bolsinov A. V., Kozlov V. V. and Fomenko A. T., The Maupertuis principle and geodesic fows on a sphere that arise from integrable case of the dynamics of a rigid body. Uspekhi Mat. Nauk, Vol. 50 (1995), pp. 3-32. English transl.: Russian Math. Surveys, Vol. 50 (1995), pp. 473-501.

[5] Selivanova E. N., New families of conservative systems on $S^{2}$ possessing an integral of fourth degree in momenta. Ann. Global Anal. Geom., 17, 201-219 (1999).

[6] Hadeler K. P. and Selivanova E. N., On the case of Kovalevskaya and new cases of integrable conservative systems on $S^{2}$. Reg. Chaot. Dyn., 4, 3, 45-52 (1999).

[7] Dullin H. R., Matveev V. S., A new integrable system on the sphere. Math. Res. Lett., 11, 10001-10008 (2004). 
[8] Ranada M., Santander M., Super-integrable systems on the two-dimensional sphere $S^{2}$ and the hyperbolic plane $H_{2}$. J. Math. Phys., 40, 5026-5057 (1999).

[9] Ranada M., Santander M., On harmonic oscillators on the two-dimensional sphere $S^{2}$ and the hyperbolic plane $\mathrm{H}_{2}$. J. Math. Phys., 43, 431-451 (2002).

[10] Yehia H. M., On the integrability of certain problems in particle and rigid body dynamics, J. Méc. Théor. Appl., Vol. 5, No. 1, (1986), pp.55-71.

[11] Yehia H. M., Two-dimensional conservative mechanical system with quartic second integral, Reg. Chaot. Dyn. , Vol. 11, (2006), pp. 103-122.

[12] Yehia H. M., Kovalevskaya's integrable case: Generalization and related new results. Reg. Chaot. Dyn. , 8,(2003), pp. 337-48.

[13] Yehia H. M., Generalized natural mechanical systems of two degrees of freedom with quadratic integrals. J. Phys A: Math. Gen., 25, 197-221 (1992).

[14] Yehia H. M., Atlas Of Two-Dimensional Irreversible Conservative Lagrangian Mechanical Systems With A Second Quadratic Integral. J. of Math. Phys. 48, 082902 (2007).

[15] Yehia H. M., On certain two-dimensional conservative mechanical systems with cubic second integral. J. Phys. A: Math. Gen., 35, 9469-9487 (2002).

[16] Yehia H. M., The Master integrable two-dimensional system with a quartic second integral. J. Phys. A: Math. Gen., 39, 5807 - 5824 (2006).

[17] Yehia H. M. and Elmandouh A. A., New conditional integrable cases of motion of a rigid body with Kovalevskaya's configuration, J. Phys. A: Math. Theor. 44 (2011) 012001 (8pp) FTC.

[18] Birkhoff G., Dynamical Systems, Amer. Math. Soc. Colloq. publ., 9, (1927).

[19] Pars L., A Treatise on Analytical Dynamics. (London: Heinemann), 1964.

[20] Lanczos C., The variational Principles Of Mechanics. Toronto Univ. Press. Toronto. 1952.

[21] Bozis G Compatibility conditions for a non-quadratic integral of motion. Celest. Mech. 28 367-80 (1982).

[22] InozemtsevV. I., Integrable models of motion of two interacting particles in the external field. J. Phys. A: Math. Gen., 17, 815-818 (1984).

[23] Wojciechowski S. 1984 On the integrability of the Calogero-Moser system in an external quartic potential and other many-body systems. Phys. Lett. A 102 85-88. 
[24] Chaplygin S. A., A new particular solution of the problem of motion of a solid in a liquid. Trudy Otdel. Phys. Nauk Obsh. Liub. Estest., 11,2, 7-10 (1903).

[25] Goriatchev D. N., New case of integrability of the Euler dynamical equations. Varshav. Univ. Izvest., No 3, 1-13 (1916). 